\title{
Edmond et Jules De Goncourt, Journal
}

\section{Ida Merello}

\section{OpenEdition}

\section{Journals}

\section{Edizione digitale}

URL: http://journals.openedition.org/studifrancesi/30656

DOI: 10.4000/studifrancesi.30656

ISSN: 2427-5856

\section{Editore}

Rosenberg \& Sellier

\section{Edizione cartacea}

Data di pubblicazione: 1 avril 2006

Paginazione: 186-187

ISSN: 0039-2944

\section{Notizia bibliografica digitale}

Ida Merello, «Edmond et Jules De Goncourt, Journal», Studi Francesi [Online], 148 (XLX | I) | 2006, online dal 30 novembre 2015, consultato il 18 avril 2021. URL: http://journals.openedition.org/studifrancesi/ 30656 ; DOI: https://doi.org/10.4000/studifrancesi.30656 


\title{
Edmond et Jules De Goncourt, Journal
}

\author{
Ida Merello
}

\section{NOTIZIA}

EDMOND ET JULES DE GONCOURT, Journal, t.I: 1851-1857, édition critique publiée sous la direction de Jean-Louis Cabanès, texte établi par Christiane et Jean-Louis Cabanès, pp. 875.

Questa nuova edizione del Journal dei Goncourt, che arriva dopo l'edizione nei Laffont, appare pienamente giustificata per il fatto di seguire tutt'altri criteri. Mentre infatti Robert Ricatte (il cui testo è stato ripreso nella collezione dei «Bouquins») praticava una collazione tra il manoscritto che gli scrittori volevano pubblicato solo dopo la morte e il testo approvato per la stampa da loro stessi, arrivando perciò talvolta a creare delle frasi nuove dall'accostamento delle due versioni, qui gli A. prendono come punto fermo soltanto il manoscritto, segnalando in nota tutte le cancellature, che spesso è difficile attribuire a un fratello piuttosto che all'altro, e mettono invece in appendice l'edizione Charpentier pubblicata da Edmond de Goncourt. M.Cabanès ricorda la difficoltà, già incontrata da Ricatte, di stabilire la punteggiatura a partire dal manoscritto, in cui essa o è assente o costituita da punti fermi che valgono un po' per tutto. Gli A. hanno cercato di non modificare i paragrafi, lasciando anche frasi oscure o ellittiche, nonché l'uso dei trattini, per quanto fosse molto più utilizzato nel XIX secolo di ora; hanno convenuto però di modernizzare la punteggiatura e corretto la grafia dei nomi propri. Ma la grande originalità e la profonda innovazione di questa edizione sta nella segnalazione all'inizio di ogni anno di quale dei fratelli sta tenendo la penna, con immediata comunicazione del cambiamento là dove questo si verifica. Questo permette di evidenziare giochi di ruolo che altrimenti non si potrebbero riconoscere (come per il caso di Jules che scrive ricordi cronologicamente attribuibili al solo Edmond) e i passaggi di mano in una descrizione. Per le note critiche gli A. si sono avvalsi di un'intera équipe di studiosi, che ha potuto meglio abbracciare i vari campi di conoscenza, creando alla fine del volume un repertorio delle informazioni biografiche 
dei personaggi menzionati, quando non sono già troppo conosciuti. Gli A. ricordano ancora come il Journal sia frutto di un montaggio di scritture diverse (pezzi di antologia e registrazioni di emozioni) avvertendo come sia sempre importante lo scarto temporale tra il moment in cui una frase è stata ascoltata, quando è stata riportata e dopo quale cosa è stata inserita nel Journal. Occorre infatti sempre tener presente come il Journal non sia una scrittura privata, ma un'opera ambiziosa, volta a rappresentare l'«ondoyante humanité dans sa vérité momentanée», mediata tuttavia da un interesse artistico palese. Per questo gli A. lo definiscono un «texte matriciel», alla base di tutti i loro romanzi e persino delle loro opere di storia dell'arte. 